\title{
Preparation, Pharmacokinetic Profile, and Tissue Distribution Studies of a Liposome-Based Formulation of SN-38 Using an UPLC-MS/MS Method
}

\author{
Kai $\mathrm{Li}^{1}$ and Shujun Wang ${ }^{1,2}$
}

\begin{abstract}
Received 11 October 2015; accepted 17 January 2016; published online 29 January 2016
Abstract. The application of 7-ethyl-10-hydroxycamptothecin ( $\mathrm{SN}-38)$ in cancer treatment is limited by its low solubility. This study is to develop a liposome-entrapped formulation of SN-38 (LE-SN38) to solve the obstacle and to evaluate its pharmacokinetic profile in dogs and tissue distribution in mice. LE-SN38 which is more likely to be suitable for large-scale production was prepared by the carrier-deposition method. An UPLC-MS/MS method was used to determinate the concentration of SN-38 in this study. LESN38 was cleared rapidly from dog plasma within $1 \mathrm{~h}$, and the $\mathrm{AUC}_{0-\infty}$ values of three dosages of LESN38 indicated an apparent dose-dependent manner. As for the distribution study, the peak of SN-38 levels in most tissues were detected within 10 min after LE-SN38 administration. In addition, concentration of SN-38 in most tissues except kidney and heart in LE-SN38 group was higher than that in irinotecan hydrochloride (CPT-11) group generally, whereas the administrated CPT-11 had 20 times dosage compared to LE-SN38. LE-SN38 was rapidly eliminated from dog plasma and manifested linear dynamics in dose range of $0.411-1.644 \mathrm{mg} / \mathrm{kg}$. The distribution behavior of $\mathrm{SN}-38$ is altered in a liposome-based delivery system. At the same time, LE-SN38 has lower toxicity compared to CPT-11 in some degree.
\end{abstract}

KEYWORDS: carrier-deposition method; LE-SN38; pharmacokinetic study; tissue distribution; UPLC-MS/MS.

\section{INTRODUCTION}

7-Ethyl-10-hydroxycamptothecin ( $\mathrm{SN}-38)$ is the biological active metabolite of irinotecan hydrochloride (CPT-11). CPT-11 has shown clinical activity against colorectal cancer and other malignances, but it just acts as a prodrug and has to be transported to its active metabolite, SN-38, by various enzyme systems (1-3). And the metabolic conversion rate is only $2-8 \%$ in spite of the fact that CPT-11 could be converted to $\mathrm{SN}-38$ in both liver and tumor tissues $(4,5)$. In addition, it not only poses significant life threatening toxicity risks but also complicates the clinical management of patients, as the transformation from CPT-11 to SN-38 is full of variable between patients (6). Moreover, SN-38 is approximately 100-1000-fold more cytotoxic than the parent drug (CPT-11) (7-9). Due to its high anticancer activity without any conversion, the direct use of SN-38 would have a very broad application prospects. And it is more possible to be recognized by doctors and patients. However, SN-38 undergoes $\mathrm{pH}$-dependent conver-

\footnotetext{
$\overline{{ }^{1} \text { Department of Pharmaceutics, School of Pharmacy, Shenyang }}$ Pharmaceutical University, 103 Wenhua Road, Shenyang, 110016, China.

${ }^{2}$ To whom correspondence should be addressed. (e-mail: 891502865@qq.com)
}

sion of pharmacologically active lactone ring to inactive carboxylate form at $\mathrm{pH}>6$ (10). And the active lactone ring has poor solubility in aqueous solutions and is practically insoluble in most pharmaceutically acceptable excipient, as it was reported that the solubility of SN-38 in water, $\mathrm{pH} 3.5$ and 7.4 buffers were assessed as $11-38,7.2$, and $36 \mu \mathrm{g} / \mathrm{ml}$, respectively (11). Hence, the further use of SN-38 in clinic is limited by its low solubility and stability, as well as its severe toxicity (12).

These drawbacks above can potentially get over by loading the drug into delivery system, particularly liposome. By using a liposome system, drug solubility could be enhanced and the toxicity of loaded anticancer agents would be reduced as well. What is more, it could also improve stability of loaded drugs by protecting the compound from in vivo conditions like acid or alkaline conditions (13). These characters are exactly what the optimization of SN-38 delivery needs. Therefore, here, it would be a wise choice to develop a liposome-based formulation of SN-38.

It is well-known that a good understanding of drugs' in vivo behavior and the proper knowledge on the distribution is vital to investigate the action mechanism and the major target sites of the product (14).

The objective of this study is to develop a liposome-based formulation of SN-38 and to evaluate its pharmacokinetic profile and biological distribution using UPLC-MS/MS. It is expected that this experiment could provide helpful information for preclinical and clinical studies. 


\section{MATERIALS AND METHODS}

\section{Materials and Animals}

SN-38 was provided by Jingmen Shuaibang Chemical Science and Technology Co., Ltd, China. Camptothecin was purchased from Sichuang Chengxin Technology Co., Ltd, China. Irinotecan hydrochloride injection was obtained from Qilu Pharmaceutical Co., Ltd, China. Soybean phospholipid was supplied by Shanghai Advanced Vehicle Technology Co., Ltd, China. Cholesterol was provided by Tianjin Concord Technology Co., Ltd, China.

The beagle dogs were purchased from the Research Institute for Laboratory Animal in Kangping (Shenyang, China; SCXK(Liao)2009-0005). And the SPF Kunming mice weighing 18-22 $\mathrm{g}$ were obtained from the Experimental Animal Center of Shenyang Pharmaceutical University (Shenyang, China; SCXK(Liao)2010-0001). All the animal experiments mentioned in this study were in accordance with the Guidelines for the Care and Use of Laboratory Animals, and they were approved by the University Ethics Committee.

\section{Preparation of Liposome-Encapsulated SN-38}

The carrier-deposition method was used for liposome-entrapped formulation of SN-38 (LE-SN38) preparation (15). Briefly, Solutol HS ${ }^{\circledR} 15$ was solubilized in anhydrous ethanol and then transferred into a round-bottom flask which contains SN-38, cholesterol, and soybean phospholipid. After dissolution, the resulting mixture and glucose powder were rotated under ultrasonic condition for uniform mixing. Then the solution was evaporated to a thin film and rehydrated with phosphate-buffered saline (PBS) containing 10\% mannitol by magnetic stirring. The hydration process was performed at $55^{\circ} \mathrm{C}$. Ten minutes later, a homogeneous dispersion was formed and immediately sonicated at $400 \mathrm{~W}$ power for $5 \mathrm{~min}$ using probe sonicator (S-4000-010, Misonix, USA), and the whole processes was carried out in ice bath. The resulting was filtered using a $0.22 \mu \mathrm{m}$ filter before being transferred into 10 $\mathrm{ml}$ vials. Lyophilization was operated by a FD-1 freeze drier, which comprised the following steps: pre-freezing at $-40^{\circ} \mathrm{C}$ for $12 \mathrm{~h}$ and then drying for $8 \mathrm{~h}$ under the pressure of $30 \mathrm{~Pa}$.

\section{Characterization of LE-SN38}

Drug entrapment efficiency was carried out on a mini-column centrifugation method $(16,17)$. Sephadex G-50 was filled to a column $(1.0 \times 10 \mathrm{~cm})$ which was applied to separate free drug from SN-38 liposome. Briefly, $0.5 \mathrm{ml}$ of the $\mathrm{SN}-38$ liposome suspension was loaded into the gel column. Subsequently, it was centrifuged for $2 \mathrm{~min}$ and then eluted by $0.5 \mathrm{ml}$ PBS and centrifuged again. The procedure was repeated twice. Then all the eluent, the solution of liposomes enveloping SN-38, was collected. After that, $0.5 \mathrm{ml}$ of the eluent and uneluted
LE-SN38 were separately diluted by methanol. The amount of SN-38 encapsulated into liposomes was determined by UV spectrophotometer.

$\mathrm{EE}(\%)=\frac{W_{\text {enveloped }}}{W_{\text {total }}} \times 100$

$W_{\text {enveloped }}$ was the concentration of SN-38 enveloped in liposomes; $W_{\text {total }}$ was the concentration of SN-38 in liposome solution.

The zeta potential and particle size of LE-SN38 were performed on a laser light scattering Zetasizer (Nano-ZS90, Malvern, UK) at $25^{\circ} \mathrm{C}$.

\section{Pharmacokinetic Studies}

The pharmacokinetic study was carried out on beagle dogs. Before the experiment day, nine of these dogs were fasted overnight but allowed free access to water. Then the dogs were randomly divided into three groups and received injections of $0.411,0.822$, and $1.644 \mathrm{mg} / \mathrm{kg}$ single dose of LESN38, respectively. The intravenous drip was conducted for about $30 \mathrm{~min}$, then $2 \mathrm{ml}$ of blood samples were collected into heparinized centrifuge tube at $5 \mathrm{~min}, 10 \mathrm{~min} 15 \mathrm{~min}, 30 \mathrm{~min}$, $1 \mathrm{~h}, 2 \mathrm{~h}, 4 \mathrm{~h}, 6 \mathrm{~h}, 8 \mathrm{~h}, 12 \mathrm{~h}$, and $24 \mathrm{~h}$ after drug administration. The plasma samples were centrifuged and then stored at $-40^{\circ} \mathrm{C}$ until determination.

\section{Tissue Distribution Studies}

The tissue distribution experiment was studied on mice. There were eight groups with six mice in each group. For the first four groups (LE-SN38 groups), $4 \mathrm{mg} / \mathrm{kg}$ of LE-SN38 was given intravenously via the tail vein, and as for the other four groups (CPT-11 groups), CPT-11 was injected at a dose of $80 \mathrm{mg} / \mathrm{kg}$. At 10, 30, 120, and $360 \mathrm{~min}$ after administration, the mice were immediately sacrificed, and organs including heart, liver, spleen, lung, kidney, and intestine were collected; then they were rinsed with saline and then stored at $-40^{\circ} \mathrm{C}$ until analysis.

\section{Sample Analysis}

\section{Sample Preparation}

Samples were thawed to room temperature before dealing with them. Then the tissues were homogenized with physiological saline at the ratio of $1: 10(\mathrm{~g} / \mathrm{ml})$ for the liver, spleen, lung, and intestine and $1: 2(\mathrm{~g} / \mathrm{ml})$ for the heart and kidney to get tissue homogenate samples. An aliquot of $100 \mu \mathrm{l}$ IS solutions $(50 \mathrm{ng} / \mathrm{mL})$ was added to $100 \mu \mathrm{l}$ of matrix sample in $1.5 \mathrm{ml}$ microcentrifuge tubes. The sample mixture was vortexed for $30 \mathrm{~s}$ at room temperature. Next, $400 \mu \mathrm{l}$ of acetonitrile (containing $0.5 \%$ acetic acid) was added and vigorously vortexed for $1 \mathrm{~min}$ (18). After centrifugation, the supernatant was extracted and transferred to another clean centrifuge tube and evaporated under $\mathrm{N}_{2}$ flow at $50^{\circ} \mathrm{C}$. Then $100 \mu \mathrm{l} 0.1 \%$ formic acid-acetonitrile (70:30, v:v) was added and vortexed for $30 \mathrm{~s}$. After being centrifuged for $10 \mathrm{~min}, 10 \mu \mathrm{l}$ of the supernatant was injected for analysis. 
Table I. The Results of Freeze-Dried by Adding Different Protective Agents to LE-SN38 $(n=3)$

\begin{tabular}{llllll}
\hline Protective agents & None & Mannitol & Sucrose & Dextran \\
\hline Appearance & -- & ++ & - & ++ & Glucose \\
Reconstitution & -- & ++ & - & + & - \\
\hline
\end{tabular}

For appearance: ++ good appearance, + good appearance with slight shrinkage at the edge, - poor appearance,,- very poor appearance. For reconstitution: + + ease, uniform size, no crystal; + large size, some crystal; - difficult to reconstitute uniformly

\section{UPLC-MS/MS Conditions}

SN-38 and CPT (IS) were separated using an ACQUITY UPLC®BEH C18 column $(2.1 \times 50 \mathrm{~mm}$, $1.7 \mu \mathrm{m}$, Waters, USA) at $30^{\circ} \mathrm{C}$. It is a gradient elution with acetonitrile (A) and water (B, containing $0.1 \%$ formic acid) as the mobile phase, and the flow rate was $0.2 \mathrm{ml} /$ min. The initial mobile phase composition was A-B (20:80, $v / v$ ) with a $0.1 \mathrm{~min}$ held and followed by a linear increase to A-B $(99: 1, v / v)$ over a $0.1 \mathrm{~min}$ period, then held for $0.8 \mathrm{~min}$, and finally returned to $\mathrm{A}-\mathrm{B}(20: 80, v / v)$ during $0.5 \mathrm{~min}$. The total time was $4 \mathrm{~min}$ with an injection $10 \mu \mathrm{l}$ for analysis (19-21).

The Waters Xevo TQD triple quadrupole mass spectrometer (Waters Corp., Milford, MA, USA) equipped with an electrospray ion source was used for mass detection. The capillary voltage, cone voltage, and desolvation temperature were $3.05 \mathrm{kV}, 47 \mathrm{~V}$, and $300^{\circ} \mathrm{C}$, respectively. Nitrogen was used as the desolvation gas $(650 \mathrm{l} / \mathrm{h})$ and cone gas $(50 \mathrm{l} / \mathrm{h})$. And the multiple reaction monitoring (MRM) was used at the transitions of $m / z, 393.3 \rightarrow 349.2$ for SN-38 and $m / z, 349.2 \rightarrow 305.3$ for IS, respectively.

The method was validated for the quantification of SN-38 in dog plasma and mice tissue samples. The calibration curves were linear within $0.5-500 \mathrm{ng} / \mathrm{ml}$ in dog plasma and within 1$500 \mathrm{ng} / \mathrm{ml}$ in mice tissues $(r=0.9974-0.9995)$. The lower limit of quantification (LLOQ) was $0.5 \mathrm{ng} / \mathrm{ml}$ in plasma and the precision was $9.53 \%$ with accuracy of $97.32 \%$. In tissue samples, the LLOQ was $1 \mathrm{ng} / \mathrm{ml}$, and its precision was ranging from 4.65 to $8.46 \%$ with accuracy from 94.9 to $105.5 \%$. The intra- and inter-day precision at three concentrations $(1,50$, and $500 \mathrm{ng} / \mathrm{ml}$ ) was less than $10 \%$. The recoveries were accessed by comparing peak area ratios of extracted QC samples with the blank plasma and blank tissue samples spiked with SN-38 after protein precipitation at the three concentrations $(1,50$, and $500 \mathrm{ng} / \mathrm{ml})$, having a result of $92.25-98.45$ and 90.33-93.64\%, respectively. The absolute matrix effect values were in the range of $94.43-105.13 \%$ for plasma and $90.67-$ $103.23 \%$ for tissue homogenate. These results indicated that there was no significant matrix effect for SN-38 in the matrix sample for this UPLC-MS/MS determination.

\section{Statistical Analysis of Data}

All measurements were taken at least in triplicate. Results were represented as mean \pm SD. The non-compartmental pharmacokinetic data were carried with Drug and Statistics 2.0 (DAS 2.0) software (Mathematical Pharmacology Professional Committee of China, Shanghai, China). Statistical analysis of the study was calculated with SPSS 11.5 software. It was considered to be statistically significant with values of $P<0.05$.

\section{RESULTS}

\section{Preparation of Liposome-Encapsulated SN-38}

The carrier-deposition method was used to prepare LESN38. The concentration of SN-38 for the optimized formulation was $1 \mathrm{mg} / \mathrm{ml}$, and the weight percent ratios of drug-tolipids and SPC to cholesterol was 1:30 and 1:6, respectively. Lyophilization was adopted to enhance the stability of LESN38 in the formulation. However, vesicle fusion and leakage of contents can appear for the liposome solution in the absence of any protective agents (22-25). In this study, freshly prepared LE-SN38 suspensions were lyophilized with different excipients to assess for cryoprotective activity. And the results were shown in Table I. The structure of the freezedrying cakes was obviously different while mixing with different protective agents. Both mannitol and dextran groups could contain integrity freeze-drying cakes, but the dextran group expressed with a larger size and some crystal. Hence, mannitol was an ideal choice.

In another study, liposomes were freeze-dried at different concentrations of mannitol, including 3, 5, 8, and $10 \%(w / v)$. It showed that $10 \%$ mannitol (lipid to mannitol ratios of $1: 3.3$ $(w / w))$ could inhibit liposomal fusion or degradation during lyophilization, and the results were assessed by the appearance, reconstitution, and the particle size of the reconstitution solution. And the entrapment efficiency has no significant change. Hence, $10 \%$ mannitol tends to be an ideal choice to effectively protect LE-SN38 during freeze-drying procedures.

The EE, particle size, and zeta potential of LE-SN38 were obtained and shown in Table II. The mean particle size

Table II. The Formulation Parameters on Physiochemical Characteristics of LE-SN38 $(n=3)$

\begin{tabular}{llcl}
\hline & Entrapment efficiency $(\%) \pm$ SD & Size \pm SD $(\mathrm{nm})$ & Zeta potential \pm SD $(\mathrm{mV})$ \\
\hline LE-SN38 before lyophilized & $91.7 \pm 4.2$ & $130 \pm 46$ & $-0.387 \pm 0.024$ \\
Lyophilized LE-SN38 (initial) & $92.1 \pm 3.7$ & $147 \pm 21$ & $-0.402 \pm 0.017$ \\
Lyophilized LE-SN38 (4 ${ }^{\circ} \mathrm{C}$ for 3 months) & $91.3 \pm 2.6$ & $134 \pm 32$ & $-0.379 \pm 0.031$ \\
\hline
\end{tabular}


Table III. Pharmacokinetic Parameters of SN-38 After i.v. Drip Administration of 0.411, 0.822, 1.644 mg $/ \mathrm{kg} \mathrm{LE-SN38} \mathrm{in} \mathrm{Dogs}(n=3)$

\begin{tabular}{|c|c|c|c|c|}
\hline \multirow{3}{*}{$\begin{array}{l}\text { Pharmacokinetic } \\
\text { parameters }\end{array}$} & \multirow[t]{3}{*}{ Unit } & \multicolumn{3}{|l|}{ Value } \\
\hline & & \multicolumn{3}{|c|}{ i.v. drip administration } \\
\hline & & $0.411 \mathrm{mg} / \mathrm{kg}$ & $0.822 \mathrm{mg} / \mathrm{kg}$ & $1.644 \mathrm{mg} / \mathrm{kg}$ \\
\hline $\mathrm{AUC}_{0-\infty}$ & $\mathrm{ng} / \mathrm{ml} \times \mathrm{h}$ & 92.53 & 165.31 & 317.19 \\
\hline $\mathrm{AUC}_{0-24 \mathrm{~h}}$ & $\mathrm{ng} / \mathrm{ml} \times \mathrm{h}$ & 67.06 & 116.42 & 233.67 \\
\hline$t_{1 / 2}$ & $\mathrm{~h}$ & 14.73 & 15.04 & 14.83 \\
\hline$V$ & $1 / \mathrm{kg}$ & 0.10 & 0.11 & 0.10 \\
\hline CL & $1 / \mathrm{h} / \mathrm{kg}$ & $0.46 \times 10^{-2}$ & $0.49 \times 10^{-2}$ & $0.51 \times 10^{-2}$ \\
\hline
\end{tabular}

$A U C$ area under the curve, $t_{1 / 2}$ half-life, $V$ apparent volume of distribution, $C L$ clearance

was $130 \pm 46 \mathrm{~nm}(<200 \mathrm{~nm})$, with an EE of $91.7 \pm 4.2 \%$ for LESN38 before lyophilized. Because zeta potential of LE-SN38 was $-0.387 \mathrm{mV}$, the low stability should be improved. Here, lyophilization might help to this situation in some degree.

\section{Pharmacokinetic Studies}

The pharmacokinetic study was performed on beagle dogs by i.v. drip administration of $0.411,0.822$, and $1.644 \mathrm{mg} /$ $\mathrm{kg}$ LE-SN38. The non-compartmental pharmacokinetic parameters resulting from DAS 2.0 software were listed in Table III.

The concentration-time profiles of SN-38 are shown in Fig. 1. According to the figure, we can infer that the plasma concentration of SN-38 was initially decreased rapidly for all three dose groups, and then equilibrium was reached at $6 \mathrm{~h}$. The primary rapid decline indicates that SN-38 might have drive away from the plasma and been distributed into the other tissues, which was confirmed by our tissue distribution study in mice.

Furthermore, we found that the fold increased in dosage ( 0.411 versus 0.822 versus $1.644 \mathrm{mg} / \mathrm{kg}$ ) led to an approximate fold increase in $\mathrm{AUC}_{0-\infty}$ (92.5 versus 165.3 versus $317.1 \mathrm{ng} / \mathrm{ml}$ $\times$ h). No significant difference appeared $(P>0.05)$ in systemic clearance and $t_{1 / 2}$ at each dose, that is to say LE-SN38 may have linear pharmacokinetics in dose range of $0.411-1.644 \mathrm{mg}$ / $\mathrm{kg}$.

In addition, during the whole study, none of the nine dogs exhibited gastrointestinal reaction, such as vomiting and diarrhea. And there was no significant difference in body weight in dogs (data not shown). This might indicate that LE-SN38 has lower toxicity compared to CPT-11. Thus, LE-SN38 may provide a potential therapeutic option for cancer treatment.

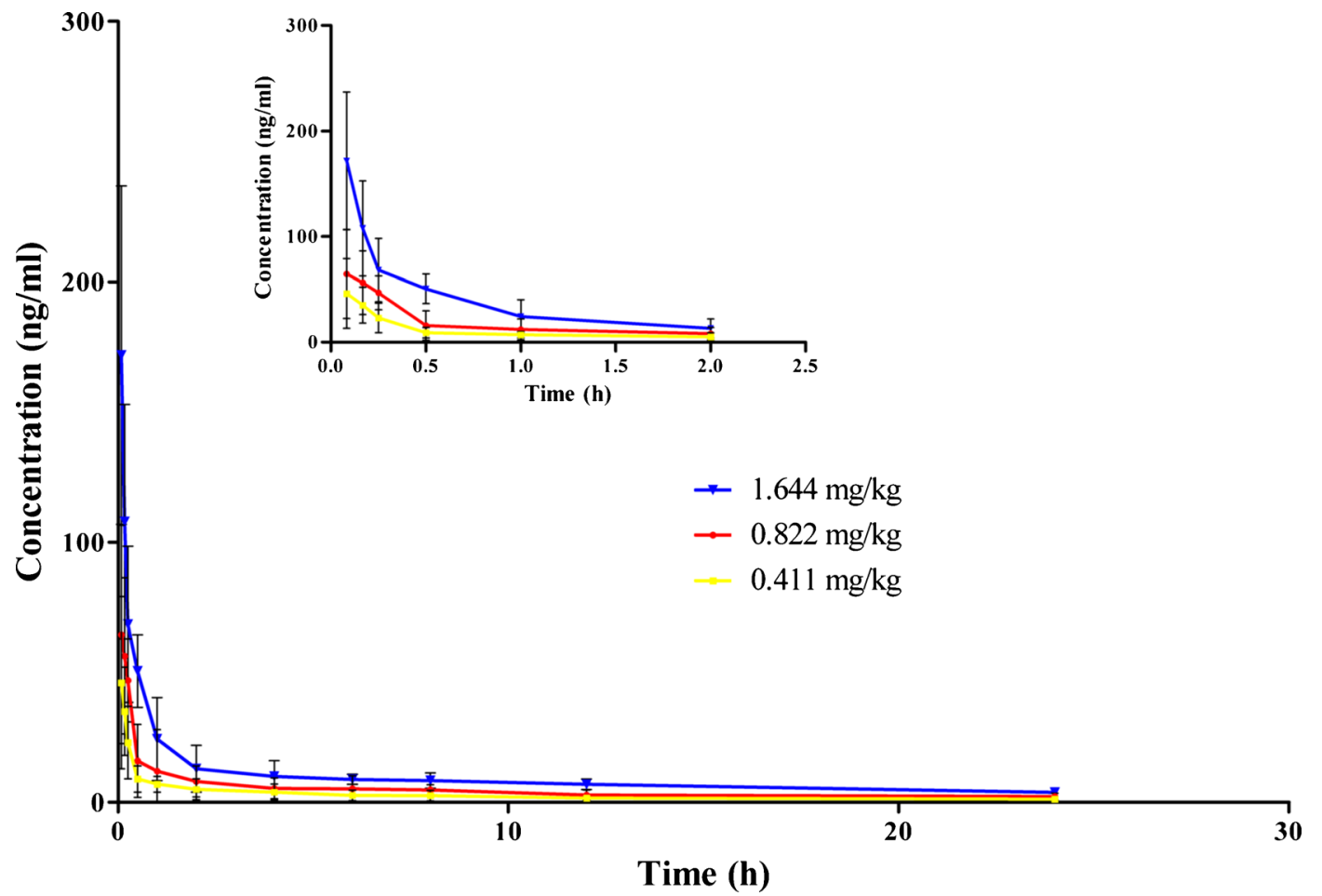

Fig. 1. Plasma concentration-time curves in beagle dogs after i.v. drip administration of $0.411,0.822$, and $1.644 \mathrm{mg} / \mathrm{kg} \mathrm{LE}-\mathrm{SN} 38(n=3)$ 


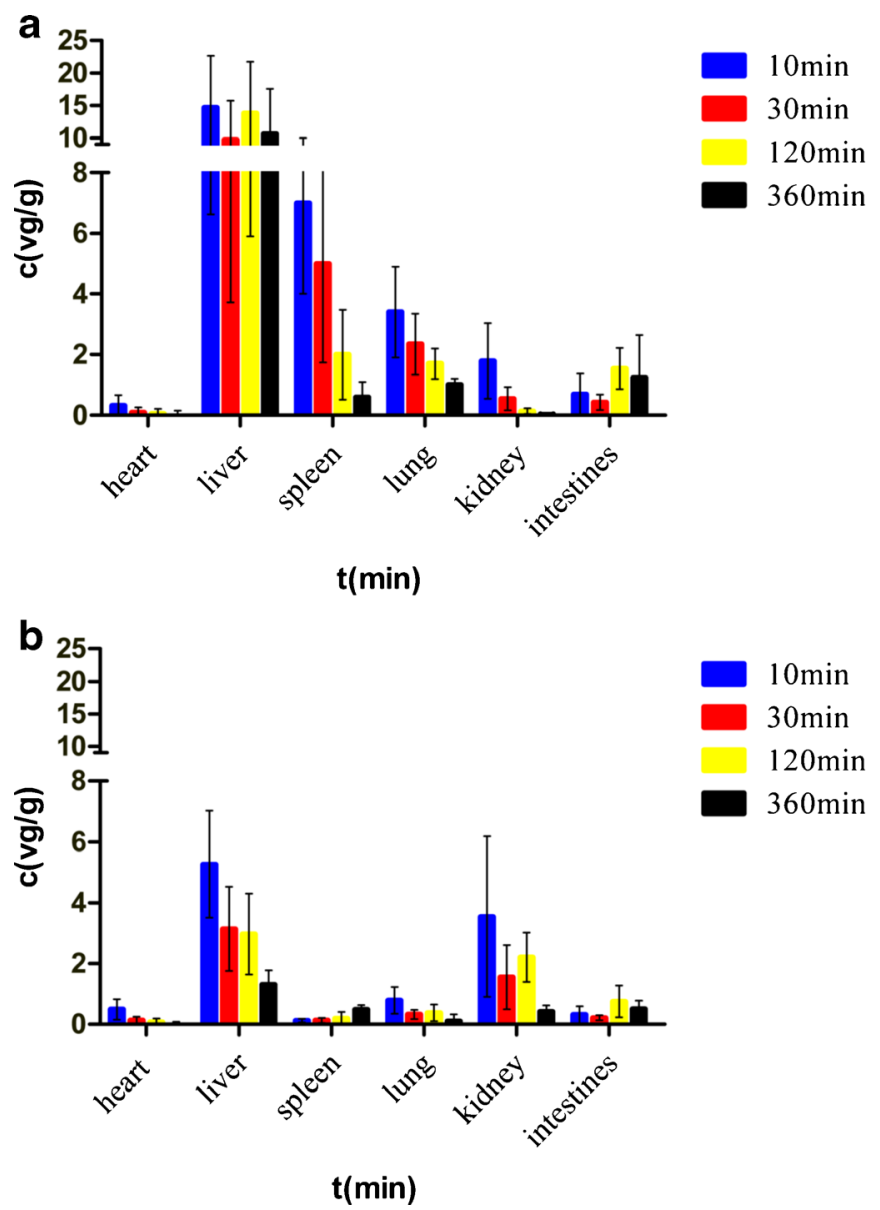

Fig. 2. Concentrations of LE-SN38 (a) and CPT-11 (b) in mice tissues at 10, 30, 120, and 360 min after i.v. administration of LE-SN38 at the dose of $4 \mathrm{mg} / \mathrm{kg}$ and CPT-11 at the dose of $80 \mathrm{mg} / \mathrm{kg}(n=6)$

\section{Tissue Distribution Studies}

Peak SN-38 levels were measured in most tissues within 10 min of treatments (Fig. 2); the results suggested that they both underwent a rapid distribution to tissues. For LE-SN38 group, the $\mathrm{AUC}_{0-6} \mathrm{~h}$ in these tissues was detected in the following order: liver $>$ spleen $>$ lung $>$ intestine $>$ kidney $>$ heart. It can be concluded that SN-38 might mainly concentrate in the liver, spleen, and lung, as they accounted for $91 \%$. While it is a wide distribution in CPT-11 group, which espe-
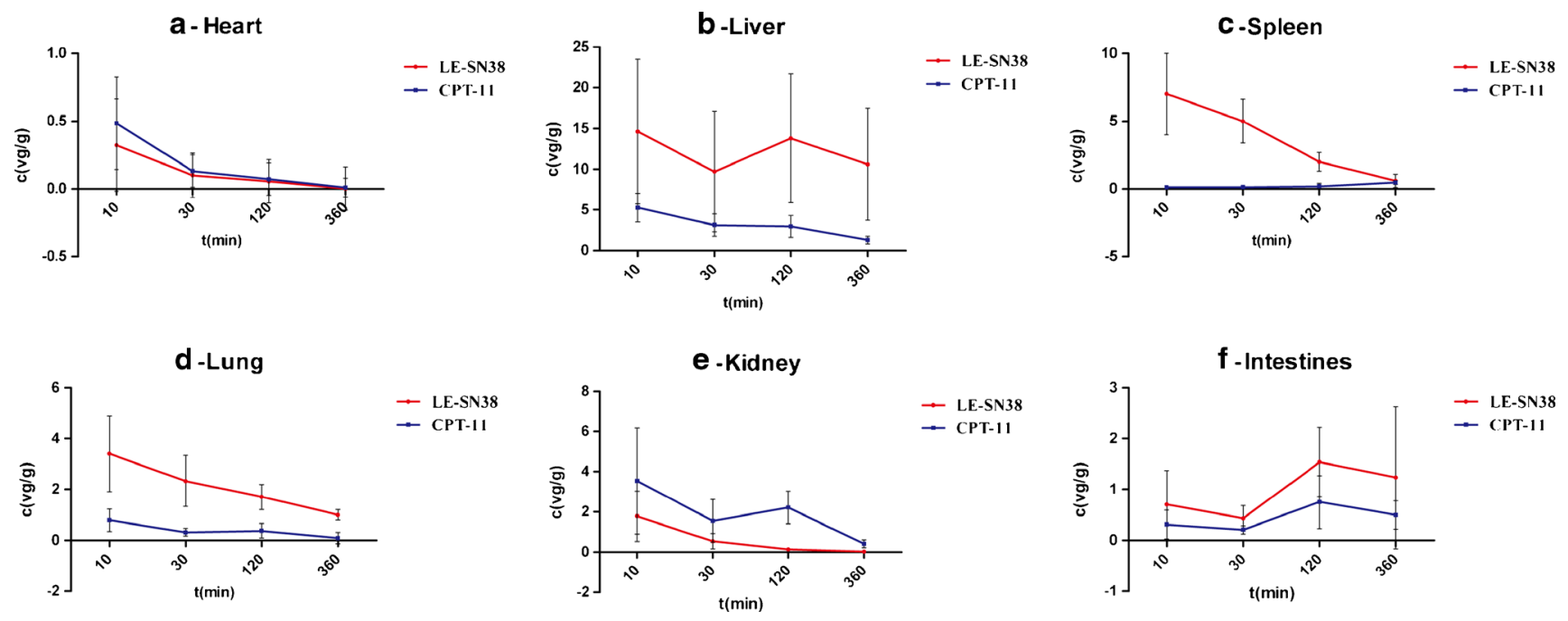

Fig. 3. a-f Tissue distribution curves of LE-SN38 and CPT- 11 in mice after i.v. administration of LE-SN38 at the dose of $4 \mathrm{mg} / \mathrm{kg}$ and CPT- 11 at the dose of $80 \mathrm{mg} / \mathrm{kg}(n=6)$ 
Table IV. AUC Values in Various Tissues After i.v. Administration of LE-SN38 (4 mg/kg) and CPT-11 $(80 \mathrm{mg} / \mathrm{kg})$ to Mice $(\mathrm{n}=6)$

\begin{tabular}{lll}
\hline Tissues & \multicolumn{2}{l}{ AUC $(\mu \mathrm{g} / \mathrm{g}$ min $)$} \\
\cline { 2 - 3 } & LE-SN38 & CPT-11 \\
\hline Heart & 18.2 & 26.3 \\
Liver & 4230.6 & 871.5 \\
Spleen & 747.1 & 97.3 \\
Lung & 564.8 & 99.7 \\
Kidney & 77.1 & 535.7 \\
Intestine & 433.7 & 200.1 \\
\hline
\end{tabular}

$A U C$ area under the curve, $L E-S N 38$ liposome-entrapped formulation of SN-38, CPT-11 irinotecan hydrochloride

cially distributed in kidney (29\%) and heart (1.4\%). And the concentrations of SN-38, with the exception of the kidney and heart, were generally higher than those following treatment with 20-fold doses of CPT-11 (Fig. 3). The $\mathrm{AUC}_{0-6 \mathrm{~h}}$ of LE-SN38 in the spleen, liver, and lung were significant higher than that of CPT-11. The $\mathrm{AUC}_{0-6} \mathrm{~h}$ for LE-SN38 was improved by $385 \%$ in the liver, $670 \%$ in the spleen, and $465 \%$ in the lung compared to CPT-11 (Table IV). These results demonstrated that LE-SN38 altered the distribution behavior of SN-38 in tissues.

\section{DISCUSSION}

In this study, LE-SN38 was prepared by carrierdeposition method, and it is a modified film dispersion method. Using this method, drug and lipids were precipitated on glucose to form solid dispersion during the evaporation. Then it can be scraped from the sides of the bottle and milled to a fine powder to obtain a more complete drying. On the other hand, glucose that is soluble in water could accelerate the hydration rate and shorten the hydration time. Moreover, the operator could timely adjust the accident occurring during the preparation (26). Therefore, the stability and quality of LE-SN38 could be enhanced by this method, and the preparation process could be more simple.

On the other hand, the product in this study would be stored in solid form as freeze-dried powder, and it leaves no contact with the hydration medium which could avoid the hydrolysis of phospholipids. Therefore, it has no physical stability and drug leakage problems which are found in the storage of liposome solution (27-30). In summary, this method solves the problem that restricted industrial production. So it is more likely to be put into large-scale production.

Recently, several methods have been reported to be applied for the quantification of SN-38 and CPT-11. But the method for determination of SN-38 in dog plasma only utilized HPLC with fluorescence detector, and it required over $6 \mathrm{~min}$ and $200 \mu \mathrm{l}$ or more plasma as sample (31-33). Hence, we would like to investigate a determination method by which shorter analysis time and lower LLOQ could be achieved. To the best of our knowledge, the bio-analytical method described here is the first UPLC-MS/MS method for dog plasma quantification of SN-38. A good separation was achieved without using any ion-pairing agents. And it has lower LLOQ
$(0.5 \mathrm{ng} / \mathrm{ml})$ in dog plasma than the previous reports determined with fluorescence detector $(1 \mathrm{ng} / \mathrm{ml})$. Moreover, it not only shortens the analysis time but also uses a smaller sample volume $(0.1 \mathrm{ml})$. These results suggest that the UPLC-MS/MS method develop here is suitable.

In the pharmacokinetic study, LE-SN38 was quickly decreasing from dog plasma. It was reported that peak $\mathrm{SN}-38$ levels were quickly measured in the liver after LE-SN38 treatment (34), and our distribution study in mice also showed that peak tissue levels were measured within $10 \mathrm{~min}$ in most tissues after LE-SN38 treatment. Furthermore, the plasma concentration in three dose groups followed the same eliminating trend. And within the dose range of $0.411-1.644 \mathrm{mg} / \mathrm{kg}$, the AUC is proportional to dose. This suggested that the safety of LE-SN38 administration is enhanced and it would also provide helpful information on clinical medicine.

On the other hand, a comparative distribution experiment was studied between LE-SN38 and CPT-11 by measuring the concentration of $\mathrm{SN}-38$ in mice tissues. Compared to CPT-11, SN-38 has relatively high drug distribution in the liver, lung, and spleen, and it still maintains a high concentration in these tissues $6 \mathrm{~h}$ later. This means that it might have good therapeutic effect for the treatment of these cancers (35), and the retention time of $\mathrm{SN}-38$ was prolonged in these organs by a liposome-based formulation. The increased uptake in the liver, lung, and spleen was probably mediated by the RES system for the characterization of liposome. Furthermore, concentration of SN-38 in the heart and kidney tended to be much lower than that of CPT-11 group, providing important evidence for reduced toxicity of LE-SN38. And this is also consistent with our pharmacokinetic study. All the results suggest that the distribution behavior of $\mathrm{SN}-38$ is altered in a liposome-based delivery system.

\section{CONCLUSION}

In this study, in order to surmount the solubility and stability obstacle of $\mathrm{SN}-38$, we have prepared a liposomebased SN-38 formulation. SN-38 lyophilized powder was prepared by carrier-deposition method, and it is stable during the storage and more possible to be put into large-scale production. Then we evaluate the pharmacokinetic profile of LESN38 in beagle dogs as well as its biological distribution in mice using an UPLC-MS/MS method. LE-SN38 was rapidly eliminated from dog plasma and followed linear pharmacokinetic pattern, and the distribution behavior of SN-38 was also altered in the liposome-based formulation. It is expected that this study could help to further clarify the in vivo behavior of LE-SN38 and provide available information for clinical application.

\section{ACKNOWLEDGMENTS}

We wish to acknowledge the support of the Pharmacy Laboratory Centre and Animal Centre of Shenyang Pharmaceutical University.

\section{COMPLIANCE WITH ETHICAL STANDARDS}

Conflict of Interest The authors declare that they have no competing interests. 


\section{REFERENCES}

1. Bardin S, Guo W, Johnson JL. Liquid chromatographic-tandem mass spectrometric assay for the simultaneous quantification of Camptosar ${ }^{\circledR}$ and its metabolite SN-38 in mouse plasma and tissues. J Chromatogr A. 2005;1073(1-2):249-55.

2. Bodurka DC, Levenback C, Wolf JK, Gano J, Wharton JT, Kavanagh JJ. Phase II trial of irinotecan in patients with metastatic epithelial ovarian cancer or peritoneal cancer. J Clin Oncol. 2003;21:291-7.

3. Saltz LB, Cox JV, Blanke C, Rosen LS, Fehrenbacher L, Moore MJ. Irinotecan plus fluorouracil and leucovorin for metastatic colorectal cancer. Irinotecan Study Group. N Engl J Med. 2000;343:905-14.

4. Ebrahimnejad P, Dinarvand R, Jafari MR. Characterization, blood profile and biodistribution properties of surface modified PLGA nanoparticles of SN-38. Int J Pharm. 2011;406:122-7.

5. Rothenberg ML, Kuhn JG, Burris HA. Phase I and pharmacokinetic trial of weekly CPT-11. Clin Oncol. 1993;11:2194-204.

6. Xuan T, Zhang JA, Ahmad I. HPLC method for determination of SN-38 content and SN-38 entrapment efficiency in a novel liposome-based formulation, LE-SN38. J Pharm Biomed Anal. 2006;41:582-8.

7. Mathijssen RH, Alphen RJV, Verweij J. Clinical pharmacokinetics and metabolism of irinotecan (CPT-11). Clin Cancer Res. 2001;7:2182-94.

8. Rowinsky EK, Grochow LB, Ettinger DS. Phase I and pharmacological study of the novel topoisomerase I inhibitor 7-ethyl-10[4-(1-piperidino)-1-piperidino]carbonyloxycamptothecin (CPT11) administered as a ninety-minute infusion every 3 weeks. Cancer Res. 1994;54:427-36.

9. Kawato Y, Aonuma M, Hirota Y, Sato K. Intracellular roles of $\mathrm{SN}-38$, a metabolite of the camptothecin derivative CPT-11, in the antitumor effect of CPT-11. Cancer Res. 1991;51:4187-91.

10. Peikov V, Ugwu S, Parmar M. pH-dependent association of SN38 with lipid bilayers of a novel liposomal formulation. Int J Pharm. 2005;299:92-9.

11. Zhang JA, Xuan T, Parmar M. Development and characterization of a novel liposome-based formulation of SN-38. Int J Pharm. 2004;270:93-107.

12. Pal A, Khan S, Wang YF. Preclinical safety, pharmacokinetics and antitumor efficacy profile of liposome-entrapped SN-38 formulation. Anticancer Res. 2005;25:331-41.

13. Xin YL, Song H, Yi J, Li YQ. Application of liposome encapsulation technique to improve anti-carcinoma effect of resveratrol. Drug Dev Ind Pharm. 2012;38(3):314-22.

14. Xie Y, Zhong GR, He HB, Fan GR, Wu YT. Determination of porcine in rat and dog plasma after intraperitoneal injection of a porcine-derived fibrin glue by fluorescein-labeled assay method: comparison with isotope-labeled assay method. J Pharm Biomed Anal. 2012;57:7-12.

15. Wang S, Ye T, Yang B. 7-Ethyl-10-hydroxycamptothecin proliposomes with a novel preparation method: optimized formulation, characterization and in-vivo evaluation. Drug Dev Ind Pharm. 2013;393-401.

16. Fry DW, White JC, Goldman ID. Rapid separation of low molecular weight solutes from liposome without dilution. J Anal Biochem. 1978;90:809-15.

17. Ye T, Jiang $\mathrm{X}$, Jing L. Low molecular weight heparin mediating targeting of lymph node metastasis based on nanoliposome and enzyme-substrate interaction. Carbohydr Polym. 2015;122:26-38.
18. Khan S, Ahmad A, Guo W. A simple and sensitive LC-MS/ MS assay for 7-ethyl-10-hydroxycamptothecin (SN-38) in mouse plasma and tissues: application to pharmacokinetic study of liposome entrapped SN-38 (LE-SN38). J Pharm Biomed. 2005;37:135-42.

19. Ramesh M, Ahlawat P, Srinivas NR. Irinotecan and its active metabolite, SN-38: review of bioanalytical methods and recent update from clinical pharmacology perspectives. Biomed Chromatogr. 2010;24(1):104-23.

20. Alexandre S, Sylvie Z, Thierry C. Metabolism of irinotecan (CPT-11) by CYP3A4 andCYP3A5 in humans. Clin Cancer Res. 2000;6:2012-2020.

21. Chen X, Peer CJ, Alfaro R. Quantification of irinotecan, SN38, and SN38G in human and porcine plasma by ultra highperformance liquid chromatography-tandem mass spectrometry and its application to hepatic chemoembolization. J Pharm Biomed. 2012;62(2):140-8.

22. Harrigan PR, Madden TD, Cullis PR. Protection of liposomes during dehydration or freezing. Chem Phys Lipids. 1990;52:13949.

23. Ye T, Xu W, Shi T. Targeted delivery of docetaxel to the metastatic lymph nodes: a comparison study between nanoliposomes and activated carbon nanoparticles. Asian J Pharm Sci. 2015;10:64-72.

24. Madden TD, Bally MB, Hope MJ. Protection of large unilamellar vesicles by trehalose during dehydration: retention of vesicle contents. BBA- Biomembr. 1985;817:67-74.

25. Wolkers WF, Oldenhof H, Tablin F. Preservation of dried liposomes in the presence of sugar and phosphate. BBA- Biomembr. 2004;1661(2):125-34.

26. Chen C, Han D, Cai C. An overview of liposome lyophilization and its future potential. J of Control Release. 2010;142:299-311.

27. Larrabee A. Time-independent changes in the distribution of distearoylphospholipid vesicles. Biochemistry. 1979;18:3321-6.

28. Wong M, Anthony FH, Tillack TW. Fusion of dipamitoylphosphatidylcholine vesicles at $4^{\circ} \mathrm{C}$. Biochemistry. 1982;21:4126-32.

29. Wong M, Thompson TE. Aggregation of dipalmitoylphospholipid vesicles. Biochemistry. 1982;21:4122-39.

30. Klein RA. The detection of oxidation in liposome preparations. Biochim Biophys Acta. 1970;210:486-9.

31. Guo W, Ahmad A, Khan S. Determination by liquid chromatography with fluorescence detection of total 7-ethyl-10-hydroxycamptothecin ( $\mathrm{SN}-38)$ in beagle dog plasma after intravenous administration of liposome-based SN-38 (LE-SN38). J Chromatogr B. 2003;791(1-2):85-92.

32. Chollet DF, Goumaz L, Renard A. Simultaneous determination of the lactone and carboxylate forms of the camptothecin derivative CPT-11 and its metabolite SN-38 in plasma by highperformance liquid chromatography. J Chromatogr B. 1998;718(1):163-75.

33. Ghazaly E, Perry J, Kitromilidou C. Development and validation of an ultra-high performance LC-MS/MS assay for intracellular SN-38 in human solid tumour cell lines: comparison with a validated HPLC-fluorescence method. J Chromatogr B. 2014;969:213-8.

34. Guo W, Ahmad A, Khan S. Determination by liquid chromatography with fluorescence detection of total 7-ethyl-10-hydroxycamptothecin (SN-38) in beagle dog plasma after intravenous administration of liposome-based SN-38 (LE-SN38). J Chromatogr B. 2001;791(1-2):85-92.

35. Cai L, Sui G, Bin W. Detection of sensitivity of lung cancer cell lines to six types of anticancer drugs. Heilongjinag Med J. 2004;10:746-9. 